\title{
PREVALÊNCIA DE INSATISFAÇÃO CORPORAL E COMPORTAMENTO ALIMENTAR DE RISCO PARA TRANSTORNOS ALIMENTARES EM JOVENS ATLETAS
}

\author{
Leonardo de Sousa Fortes \\ Universidade Federal de Juiz de Fora, Juiz de Fora, Minas Gerais, Brasil \\ Sebastião de Sousa Almeida \\ Universidade de São Paulo, Ribeirão Preto, São Paulo, Brasil \\ Maria Elisa Caputo Ferreira \\ Universidade Federal de Juiz de Fora, Juiz de Fora, Minas Gerais, Brasil
}

\begin{abstract}
Resumo
O objetivo do estudo foi comparar a prevalência de Insatisfação Corporal (IC) e Comportamento Alimentar Inadequado (CAI) em jovens atletas. Participaram 580 atletas de ambos os sexos. Avaliou-se IC pelo Body Shape Questionnaire. Examinou-se o CAI pelo Eating Attitudes Test. Utilizou-se o qui-quadrado para comparar as prevalências entre sexos e esportes. Os resultados evidenciaram que Basquetebol e Judô foram modalidades que apresentaram mais atletas masculinos com IC $(\mathrm{p}<0,05)$. Nas meninas, estas diferenças foram significativas entre alguns esportes $(\mathrm{p}<0,05)$. Ademais, os meninos que praticavam futebol, basquetebol e tae-kwon-do demonstraram maior prevalência de CAI $(\mathrm{p}<0,05)$. Concluiu-se que existem diferenças de prevalência de IC e CAI entre os esportes, segundo o sexo.
\end{abstract}

Palavras-chave: Transtornos Alimentares. Imagem corporal. Atletas.

\section{Introdução}

A imagem corporal é um construto multifacetado e dinâmico (CONTI; CORDÁS; LATORRE, 2009). Pesquisadores têm despertado o interesse em avaliar facetas deste fenômeno (SCHERER et al., 2010), dentre elas, a insatisfação corporal (MARTINS et al., 2010), um subcomponente da dimensão atitudinal da imagem corporal que diz respeito à depreciação do indivíduo com sua aparência física (CONTI; CORDÁS; LATORRE, 2009). Estudos realizados com adolescentes brasileiros mostram prevalência de insatisfação com o corpo variando de $40 \%$ a $60 \%$ (SCHERER et al., 2010). 
Em atletas, este fenômeno parece apresentar níveis diferentes, variando entre 10\% e 30\% (FORTES et al., 2011; VIEIRA et al., 2006; VIEIRA et al., 2009b).

São considerados comportamentos alimentares inadequados: restrição alimentar por longos períodos, vômitos autoinduzidos e uso de laxantes/diuréticos para perda de peso (SCHAAL et al., 2011). Vários fatores influenciam tais hábitos, dentre os quais, insatisfação corporal (SCHERER et al., 2010). Parece que a prevalência destes comportamentos é alta no âmbito esportivo (PERINI et al., 2009), principalmente no sexo feminino (VIEIRA et al., 2009a). No entanto, variáveis comportamentais não têm sido frequentemente avaliadas no sexo masculino (BAUM, 2006). Autores recomendam que as análises de variáveis afetivas e comportamentais sejam realizadas separadas por sexo (TORSTVEIT; SUNDGOT-BORGEN, 2005; FORTES; FERREIRA, 2011).

Tanto o comportamento alimentar inadequado, quanto a insatisfação corporal são considerados sintomas de desencadeamento dos Transtornos Alimentares (TAs) (BAUM, 2006). Os TAs mais comumente encontrados na população em geral são a Anorexia Nervosa (AN) e a Bulimia Nervosa (BN). A AN inicia-se preponderantemente na adolescência, ocorrendo entre os 10 e 20 anos de idade (PERINI et al., 2009). Caracteriza-se pela restrição alimentar autoimposta, que resulta em extrema perda de massa corporal - cerca de $25 \%$ em relação à massa inicial, podendo chegar a 50\% - decorrente do intenso desejo de emagrecer, havendo distorção da imagem corporal, além de disfunções do ciclo menstrual em meninas (TORSTVEIT; SUNDGOT-BORGEN, 2005). Sujeitos com BN apresentam hábitos como grande e rápida ingestão de alimentos com sensação de perda de controle, acompanhada de métodos compensatórios inadequados para o controle da massa corporal, tais como: indução de vômitos; uso indevido de medicamentos; dietas severas e exercícios físicos extenuantes (SCHAAL et al., 2011). A prevalência destas síndromes na população geral é de $1 \%$ para $\mathrm{AN}$ e $4 \%$ para $\mathrm{BN}$ (SCHERER et al., 2010). Estudos apresentam inconsistência de ocorrência destes transtornos em atletas (FILAIRE et al., 2007; SCHAAL et al., 2011). No Brasil, até o momento, não foi encontrada nenhuma investigação que tenha comparado a prevalência de comportamentos de riscos para estas psicopatologias entre atletas de diferentes modalidades esportivas.

Evidências sugerem que esportistas competitivos possuem maior risco para desenvolvimento dos TAs (SUNDGOT-BORGEN; TORSTVEIT, 2004; DENOMA et al., 2009). Existem características típicas de alguns esportes que podem ser agentes potencializadores para tal desfecho e, entre estas, destacam-se: ênfase na magreza (TORSTVEIT; SUNDGOT-BORGEN, 2005); uniformes e roupas esportivas que salientam a forma corporal (FORTES et al., 2011); pressão de técnicos, familiares e treinadores para perda ou manutenção de peso; além da modalidade esportiva praticada (PERINI et al., 2009). Segundo Krentz e Warschburger (2011), esportes que preconizam baixa adiposidade corporal, estética voltada para a magreza e valorização da leveza dos movimentos como critérios, visando o bom de-

Pensar a Prática, Goiânia, v. 17, n. 1, p. 01-294, jan./mar. 2014 
sempenho atlético, podem ser consideradas modalidades de risco para o desencadeamento dos TAs.

Neste sentido, a participação em esportes como: nado sincronizado, saltos ornamentais, esgrima e ginástica artística pode aumentar a suscetibilidade para os TAs (FORTES; FERREIRA, 2011). Adicionalmente, Schaal et al. (2011) salientam que atletas de modalidades esportivas com divisões por classe de peso corporal, como o tae-kwon-do e o judô, também podem demonstrar maior vulnerabilidade para o desencadeamento dos TAs.

A maioria dos estudos realizados com atletas utilizou amostras do sexo feminino, pois esta população é considerada a de maior risco na instalação dos TAs (PERINI et al., 2009). Poucos estudos sobre riscos para TAs têm sido realizados com atletas do sexo masculino (BAUM, 2006), principalmente no Brasil (FORTES; FERREIRA, 2011; FORTES et al., 2011). Ademais, ainda não foi testado se existe esporte que apresente maior prevalência para insatisfação corporal e comportamento alimentar inadequado.

Diante das considerações acima descritas, o objetivo do presente estudo foi comparar a prevalência de insatisfação corporal e de comportamento alimentar inadequado em jovens atletas de ambos os sexos, galgando identificar modalidades que possam ser consideradas de risco para o desencadeamento dos TAs.

\section{Método}

Aspectos éticos

O estudo obteve aprovação do Comitê de Ética e Pesquisa Envolvendo Seres Humanos da Universidade Federal de Juiz de Fora com o parecer número 232/2010, de acordo com a resolução 196/96. O Termo de Consentimento Livre e Esclarecido foi assinado pelos pais dos atletas.

\section{Amostra}

A população do estudo foi constituída por atletas competitivos adolescentes, com idades entre 10 e 19 anos, residentes em cidades dos Estados brasileiros do Rio de Janeiro/RJ e Minas Gerais/MG. Com base no Comitê Olímpico Brasileiro, esta população era na ordem de 24.252 indivíduos, no ano de 2010.

O tamanho da amostra foi calculado considerando prevalência de comportamentos alimentares de risco para TAs de $20 \%$, com $95 \%$ de confiança, $4 \%$ de erro amostral, e efeito de desenho de 1,4, totalizando 379 jovens. A prevalência adotada para o cálculo amostral (20\%) foi uma aproximação dos valores encontrados em dois estudos nacionais (PERINI et al., 2009; VIEIRA et al., 2009b). Inicialmente, foram incluídos na pesquisa 620 atletas de ambos os sexos. Porém, 40 destes sujeitos foram excluídos da amostra por não responderem os questionários em 
sua totalidade, o que resultou em uma amostra final de 580 atletas (464 meninos e 116 meninas), com média de 14,95 \pm 2 anos para idade, praticantes de diversas modalidades (atletismo, basquetebol, esgrima, futebol, ginástica artística, handebol, judô, nado sincronizado, natação, polo aquático, saltos ornamentais, tae-kwon-do, triathlon e voleibol).

Para inclusão de sujeitos na pesquisa, foram adotados os seguintes critérios:

1. Apresentar o TCLE assinado pelo responsável;

2. Estar em processo de treinamento físico sistematizado de sua respectiva modalidade esportiva, com frequência mínima de três dias semanais e duração mínima de $1 \mathrm{~h} /$ treino;

3. Ter participado de pelo menos uma competição de dimensão regional no ano de 2011;

4. Ter disponibilidade para responder questionários.

Instrumentos

Para avaliar a insatisfação corporal foi aplicado o Body Shape Questionnaire (BSQ). Trata-se de um teste de autopreenchimento com 34 perguntas que procuram avaliar a preocupação que o sujeito apresenta com o seu peso e com a sua aparência física. A versão utilizada foi validada para adolescentes brasileiros (CONTI; CORDÁS; LATORRE, 2009). Para a presente amostra, foram utilizados valores do $\alpha$ de Cronbach de 0,91 para as meninas e 0,92 para os meninos. A pontuação do BSQ é dada pela soma de seus itens, que classifica níveis de insatisfação a respeito do corpo, sendo: $<80$ pontos $=$ livre de insatisfação corporal, e $\geq 80$ pontos $=$ algum nível de insatisfação corporal.

Para avaliar o comportamento alimentar inadequado foi aplicado o Eating Attitudes Test (EAT-26). Este é um questionário composto de 26 questões que avaliam: recusa alimentar patológica, preocupação exacerbada com aparência física, comportamentos purgativos, influência do ambiente na ingestão alimentar e autocontrole sobre os alimentos. A versão utilizada foi validada para adolescentes brasileiros do sexo feminino (BIGHETTI et al., 2004) e masculino (FORTES et al., no prelo).

No estudo de Fortes et al. (no prelo), os autores não encontraram diferença no teste-reteste, evidenciaram validade discriminante e concorrente do EAT-26, além de identificarem consistência interna de 0,87 avaliada pelo Alpha de Cronbach. Calculou-se o $\alpha$ de Cronbach para o presente estudo, identificando-se valores de 0,89 e 0,92 para meninas e meninos, respectivamente. A pontuação do EAT26 é feita pela soma dos itens. Pontuação igual ou maior que 20 representa indivíduos com comportamento alimentar de risco para TAs (EAT+ - "positivo").

Por fim, foi aplicado um questionário qualitativo para avaliar dados demográficos: idade, sexo e tipo de esporte. 


\section{Procedimentos}

Foi realizado um encontro com todos os atletas. Este foi destinado à aplicação dos questionários BSQ e EAT-26. Todos os clubes que fizeram parte do presente estudo disponibilizaram uma sala adequada para a realização da atividade. Os questionários foram entregues aos atletas, que receberam a mesma orientação verbal. Havia também uma orientação escrita sobre os procedimentos adequados. Os sujeitos do estudo não se comunicaram entre si. A distribuição dos instrumentos foi efetuada no momento em que os atletas adentravam no ambiente (sala), sendo seu preenchimento de caráter voluntário. Não houve limite de tempo estabelecido.

Análise dos dados

O qui-quadrado de uma variável foi aplicado para avaliar diferenças de prevalência de insatisfação corporal e comportamento alimentar entre os sexos. Para isto, as classificações de insatisfação corporal (leve insatisfação, moderada insatisfação e grave insatisfação) do BSQ foram agrupadas como "insatisfeito". Este mesmo teste foi utilizado para identificar diferenças na prevalência de comportamento alimentar inadequado e insatisfação corporal entre modalidades esportivas, em função do sexo. Todos os dados foram analisados no software SPSS 17.0 com nível de significância de $5 \%$.

\section{Resultados}

A comparação da prevalência de insatisfação corporal entre sexos, em atletas adolescentes brasileiros está ilustrada na Figura 1. Foi identificada diferença de frequência de insatisfação corporal entre os sexos $(p<0,05)$. 




Figura 1 - Comparação de prevalência de insatisfação corporal entre sexos, em jovens atletas brasileiros

$* \mathrm{p}<0,05$

Na Tabela 1 estão elucidadas comparações de prevalência de insatisfação corporal entre modalidades esportivas do sexo masculino. Basquetebol (25\%) e Judô (29\%) foram modalidades que apresentaram maior frequência de atletas insatisfeitos com o corpo $(\mathrm{p}<0,05)$. No sexo feminino, foram encontradas diferenças estatisticamente significativas de prevalência deste fenômeno entre alguns esportes $(\mathrm{p}<0,05)$ (Tabela 2). 
Tabela 1 - Comparação de prevalência (\%) de insatisfação corporal entre modalidades es portivas do sexo masculino

\begin{tabular}{|c|c|c|}
\hline Modalidade / Insatisfação Corporal & Satisfeito & Insatisfeito \\
\hline Natação ${ }^{b, c, d, e, f, g, h, i, j, 1}$ & 93 & 7 \\
\hline Futebol a, c, d, e, g, h, i, j, k, 1 & 84 & 16 \\
\hline Handebol a, b, d, f, h, k & 100 & 0 \\
\hline Basquetebol a, b, c, e, g, i, j, k, l & 75 & 25 \\
\hline Voleibol a, b, d, f, h, k & 100 & 0 \\
\hline 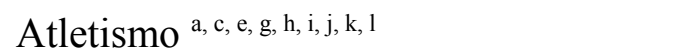 & 80 & 20 \\
\hline Triathlon $^{\mathrm{a}, \mathrm{b}, \mathrm{d}, \mathrm{f}, \mathrm{h}, \mathrm{k}}$ & 100 & 0 \\
\hline 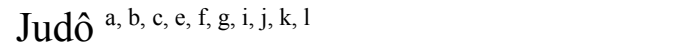 & 71 & 29 \\
\hline Tae-kwon-do $\mathrm{b}, \mathrm{d}, \mathrm{f}, \mathrm{h}, \mathrm{k}$ & 100 & 0 \\
\hline Esgrima $^{\mathrm{a}, \mathrm{b}, \mathrm{d}, \mathrm{f}, \mathrm{h}, \mathrm{k}}$ & 100 & 0 \\
\hline Polo Aquático b, c, d, e, f, g, h, i, j, k, 1 & 94 & 6 \\
\hline Saltos Ornamentais ${ }^{\mathrm{a}, \mathrm{b}, \mathrm{d}, \mathrm{f}, \mathrm{h}, \mathrm{k}}$ & 100 & 0 \\
\hline \multicolumn{3}{|c|}{$\begin{array}{l}{ }^{a} \mathrm{p}<0,05 \text { em relação à Natação; }{ }^{b} \mathrm{p}<0,05 \text { em relação ao Futebol; }{ }^{c} \mathrm{p}<0,05 \text { em relação ao } \\
\text { Handebol; }{ }^{\mathrm{p}} \mathrm{p}<0,05 \text { em relação ao Basquetebol; }{ }^{\mathrm{e}} \mathrm{p}<0,05 \text { em relação ao Voleibol; }{ }^{\mathrm{p}}<0,05 \\
\text { em relação ao Atletismo; }{ }^{\mathrm{g}} \mathrm{p}<0,05 \text { em relação ao Triathlon; }{ }^{\mathrm{h}} \mathrm{p}<0,05 \text { em relação ao Judô; } \\
\mathrm{i} \mathrm{p}<0,05 \text { em relação ao Tae-kwon-do; }{ }^{\mathrm{p}} \mathrm{p}<0,05 \text { em relação à Esgrima; }{ }^{\mathrm{k}} \mathrm{p}<0,05 \text { em relação } \\
\text { ao Pólo Aquático; }{ }^{1} \mathrm{p}<0,05 \text { em relação aos Saltos Ornamentais. }\end{array}$} \\
\hline
\end{tabular}

Tabela 2 - Comparação de prevalência (\%) de insatisfação corporal entre modalidades es portivas do sexo feminino

\begin{tabular}{lcc}
\multicolumn{1}{c}{ Modalidade / Insatisfação Corporal } & Satisfeita & Insatisfeita \\
\hline Natação $^{\text {d, f, g, h }}$ & 54,5 & 45,5 \\
Handebol $^{\text {d, e, f, g, h }}$ & 50 & 50 \\
Basquetebol $^{\text {d, e, f, , , h }}$ & 50 & 50 \\
Voleibol $^{\text {a, b, c, e, h }}$ & 75 & 25 \\
Tae-kwon-do $^{\text {c, d, e, f, h }}$ & 60 & 40 \\
Ginástica Artística $^{\text {a, b, c, e, h }}$ & 71 & 29 \\
Nado Sincronizado $^{\text {a, b, c, d, h }}$ & 67 & 33 \\
Saltos Ornamentais $^{\text {a, b, c, e, g }}$ & 80 & 20 \\
\hline
\end{tabular}

${ }^{a} p<0,05$ em relação à Natação; ${ }^{b} p<0,05$ em relação ao Handebol; ${ }^{c} p<0,05$ em relação ao Basquetebol; ${ }^{\mathrm{d}} \mathrm{p}<0,05$ em relação ao Voleibol; ${ }^{\mathrm{e}} \mathrm{p}<0,05$ em relação ao Tae-kwon-do; ${ }^{\mathrm{f}} \mathrm{p}<0,05$ em relação à Ginástica Artística; ${ }^{\mathrm{g}} \mathrm{p}<0,05$ em relação ao Nado Sincronizado ${ }^{h} \mathrm{p}<0,05$ em relação aos Saltos Ornamentais.

A Figura 2 apresenta a comparação da prevalência de comportamento alimentar inadequado entre sexos, em jovens atletas. Não houve diferença de prevalência entre estes. 


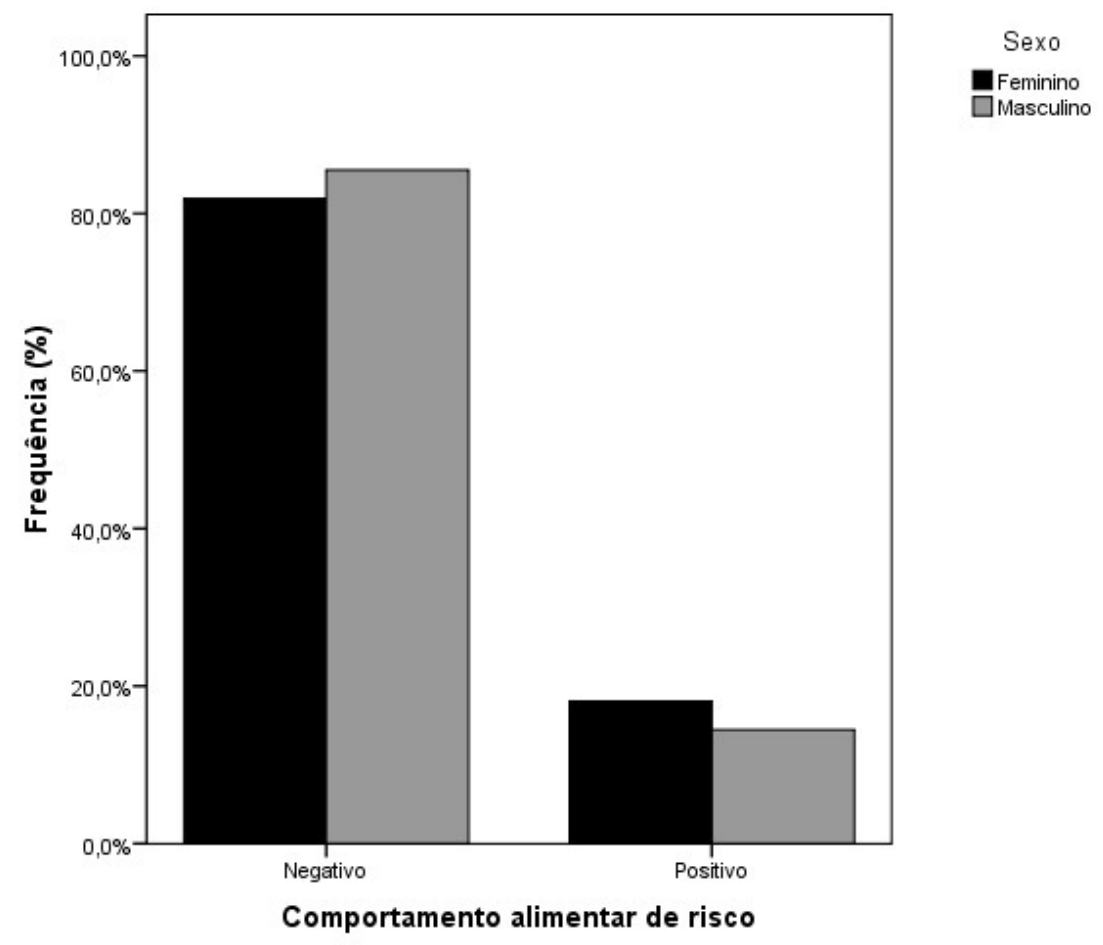

Figura 2 - Comparação de prevalência de comportamento alimentar de risco entre sexos, em jovens atletas brasileiros

As Tabelas 3 e 4 apresentam comparações de prevalência de comportamento alimentar de risco para TAs entre modalidades esportivas do sexo masculino e feminino, respectivamente. Meninos que praticavam futebol, basquetebol e tae-kwon-do apresentaram maior prevalência para tais riscos $(\mathrm{p}<0,05)$. Em atletas do sexo feminino, apenas esportes como voleibol e saltos ornamentais apresentaram $0 \%$ de prevalência na classificação "positivo" do EAT-26, e as demais modalidades esportivas demonstraram prevalências semelhantes de comportamento alimentar inadequado. 
Tabela 3 - Diferença de prevalência (\%) de comportamento alimentar de risco para TAs entre modalidades esportivas do sexo masculino

\begin{tabular}{|c|c|c|}
\hline Modalidade / EAT-26 & Negativo $(<20)$ & Positivo $(\geq 20)$ \\
\hline Natacãão ${ }^{d, e, g, j, k, 1}$ & 88 & 12 \\
\hline Futebol e, g, h, j, k, 1 & 83 & 17 \\
\hline Handebol ${ }^{\mathrm{d}, \mathrm{e}, \mathrm{g}, \mathrm{j}, \mathrm{k}, 1}$ & 87 & 13 \\
\hline Basquetebol a, e, f, g, h, j, k, 1 & 80 & 20 \\
\hline Voleibol $^{\mathrm{a}, \mathrm{b}, \mathrm{c}, \mathrm{d}, \mathrm{f}, \mathrm{h}, \mathrm{i}}$ & 100 & 0 \\
\hline Atletismo ${ }^{\mathrm{d}, \mathrm{e}, \mathrm{f}, \alpha, \mathrm{j}, \mathrm{k}, 1}$ & 90 & 10 \\
\hline Triathlon ${ }^{\mathrm{a}, \mathrm{b}, \mathrm{c}, \mathrm{d}, \mathrm{f}, \mathrm{h}, \mathrm{i}}$ & 100 & 0 \\
\hline Judô ${ }^{b, d, e, g, a, j, k, 1}$ & 93 & 7 \\
\hline Tae-kwon-do ${ }^{\mathrm{h}, \mathrm{j}, \mathrm{k}, 1}$ & 83 & 17 \\
\hline Esgrima a, b, c, d, f, h, i, k & 100 & 0 \\
\hline Polo Aquático a, b, c, d, f, h, i & 100 & 0 \\
\hline Saltos Ornamentais $\mathrm{a}, \mathrm{b}, \mathrm{c}, \mathrm{d}, \mathrm{f}, \mathrm{h}, \mathrm{i}$ & 100 & 0 \\
\hline
\end{tabular}

${ }^{a} \mathrm{p}<0,05$ em relação à Natação; ${ }^{b} \mathrm{p}<0,05$ em relação ao Futebol; ${ }^{\mathrm{c}} \mathrm{p}<0,05$ em relação ao Handebol; ${ }^{d} \mathrm{p}<0,05$ em relação ao Basquetebol; ${ }^{\mathrm{e}} \mathrm{p}<0,05$ em relação ao Voleibol; ${ }^{\mathrm{p}}<0,05$ em relação ao Atletismo; ${ }^{\mathrm{p}} \mathrm{p}<0,05$ em relação ao Triathlon; ${ }^{\mathrm{h}} \mathrm{p}<0,05$ em relação ao Judô; ${ }^{\mathrm{i}} \mathrm{p}<0,05$ em relação ao Tae-kwon-do; ${ }^{\mathrm{j}} \mathrm{p}<0,05$ em relação à Esgrima; ${ }^{\mathrm{k}} \mathrm{p}<0,05$ em relação ao Pólo Aquático; ${ }^{1} \mathrm{p}<0,05$ em relação aos Saltos Ornamentais.

Tabela 4 - Diferença de prevalência (\%) de comportamento alimentar de risco para TAs entre modalidades esportivas do sexo feminino

\begin{tabular}{|c|c|c|}
\hline Modalidade / EAT-26 & Negativo $(<20)$ & Positivo $(\geq 20)$ \\
\hline Natação ${ }^{\mathrm{d}, \mathrm{h}}$ & 77 & 23 \\
\hline Handebol ${ }^{\mathrm{d}, \mathrm{h}}$ & 80 & 20 \\
\hline Basquetebol $^{\mathrm{d}, \mathrm{h}}$ & 75 & 25 \\
\hline Voleibol a, b, c, e, f, g & 100 & 0 \\
\hline Tae-kwon-do ${ }^{\mathrm{d}, \mathrm{h}}$ & 80 & 20 \\
\hline Ginástica Artística $^{\mathrm{d}, \mathrm{h}}$ & 79 & 21 \\
\hline Nado Sincronizado $^{\mathrm{d}, \mathrm{h}}$ & 78 & 22 \\
\hline Saltos Ornamentais ${ }^{a, b, c, e, f, g}$ & 100 & 0 \\
\hline
\end{tabular}

\section{Discussão}

Este estudo buscou comparar a prevalência de insatisfação corporal e comportamento alimentar inadequado entre jovens atletas brasileiros. Os resultados evidenciaram prevalência semelhante de insatisfação corporal e altas frequências de comportamento alimentar inadequado em ambos os sexos, quando comparadas 
aos resultados de pesquisas envolvendo amostras com adolescentes escolares (CONTI; CORDÁS; LATORRE, 2009; SCHERER et al., 2009). No entanto, foi encontrada diferença entre os sexos para prevalência de insatisfação corporal. Ademais, identificou-se diferenças de prevalência de insatisfação corporal e comportamento alimentar inadequado entre modalidades esportivas em ambos os sexos.

Os resultados relativos à aplicação do BSQ corroboram outros estudos nacionais conduzidos com atletas adolescentes (PERINI et al., 2009; FORTES; FERREIRA, 2011), e demonstram maior prevalência de indivíduos satisfeitos com o corpo quando comparada à população adolescente em geral (SCHERER et al., 2010). Em pesquisas com atletas de outros países, esta tendência também foi confirmada (HAUNSENBLAS; DOWNS, 2001; FILAIRE et al., 2007).

Adolescentes do sexo feminino, independentemente de serem atletas ou não, são mais insatisfeitas com o corpo que os meninos da mesma faixa etária (SUNDGOT-BORGEN; TORSTVEIT, 2004). Os resultados do presente estudo confirmam esta afirmação, pois foi identificada maior prevalência de insatisfação com o corpo em atletas femininas, quando comparadas com indivíduos do sexo masculino. Contudo, salienta-se que o BSQ se propõe a avaliar somente uma faceta da insatisfação corporal, ou seja, a depreciação associada à gordura corporal. Deste modo, questões como sentimentos a respeito da muscularidade, item fulcral da imagem corporal masculina (FORTES et al., 2011), não foram comparados entre os sexos, caracterizando-se como uma limitação da presente investigação.

Neste estudo, encontrou-se maior prevalência de insatisfação corporal no judô (29\%), basquetebol (25\%), atletismo (20\%) e futebol (16\%), no sexo masculino. Estudos não têm comparado a prevalência de insatisfação com a aparência física em atletas de diferentes esportes, com características distintas. Além disso, atletas do sexo masculino não têm sido frequentemente avaliados em relação à insatisfação corporal.

Em um dos poucos estudos com homens, Pickett, Lewis e Cash (2005), avaliaram a insatisfação corporal de atletas bodybuilders, levantadores de peso, basquetebolistas, corredores e lutadores de artes marciais que praticavam treinamento físico sistematizado de suas respectivas modalidades pelo menos três vezes por semana. Estes autores identificaram que bodybuilders e levantadores de peso estavam mais satisfeitos com o peso e a aparência corporal que o restante dos atletas. Isso nos remete a pensar que atletas do sexo masculino de esportes com características eminentemente estéticas podem ter imagem corporal mais positiva do que atletas de modalidades de resistência, classe de peso/combate e coletiva.

Já entre as meninas, as modalidades com maiores frequências de insatisfação corporal foram: basquetebol (50\%), handebol (50\%), natação $(45,5 \%)$, taekwon-do (40\%), e nado sincronizado (33\%). Estes resultados corroboram com a hipótese de que, independentemente das características da modalidade (estética, potência, divisão por classe de peso, etc), existe elevada prevalência de insatisfa- 
ção com o peso e a aparência física em atletas femininas (KRENTZ; WARSCHBURGER, 2011).

Entretanto, existem resultados controversos, pois nos estudos de Perini et al. (2009) e Vieira et al. (2009a), na avaliação de atletas de nado sincronizado e ginástica rítmica, respectivamente, foram encontradas baixas prevalências de insatisfação corporal. Por outro lado, Reiking e Alexander (2005), em seus estudos, identificaram maior prevalência de insatisfação corporal em atletas de esportes de magreza.

A insatisfação corporal, segundo Perini et al. (2009), é sintoma de primeira ordem no desencadeamento de TAs. No entanto, este fenômeno em atletas parece mostrar características distintas, pois em algumas investigações (DENOMA et al., 2009; VIEIRA et al., 2009b), autores identificaram baixa prevalência de insatisfação corporal. Por outro lado, encontraram altas frequências de comportamento alimentar inadequado. Este fato pode ocorrer devido à pressão sofrida pelos atletas, exercida por pais, treinadores e patrocinadores no anseio por melhores resultados (SUNDGOT-BORGEN; TORSTVEIT, 2004), além da exigência aos esportistas quanto ao controle de peso, fato que pode vir a estimular a aquisição de comportamentos alimentares inapropriados (BAUM, 2006). Essa autora afirma que o treinamento voltado para a performance pode ser considerado como um impacto negativo para a gênese do comportamento alimentar de risco em atletas.

Os resultados do presente estudo vão ao encontro dos dados apresentados pela literatura científica (HAUSENBLAS; DOWNS, 2001; FILAIRE et al., 2007), já que não foi identificada diferença estatisticamente significativa de prevalência de comportamento alimentar inadequado entre os sexos. Pesquisas têm demonstrado que o sexo feminino apresenta maior frequência de hábitos alimentares não saudáveis (SCHAAL et al., 2011). Talvez, este fenômeno ocorra pelo fato de meninas parecerem sofrer maior pressão cultural a respeito do ideal de magreza (FORTES; FERREIRA, 2011), sendo incentivadas a adquirirem comportamentos alimentares considerados patológicos na tentativa de perda/controle do peso corporal (SCHERER et al., 2010). Ademais, autores salientam que, no âmbito esportivo, o sexo feminino costuma absorver negativamente as cobranças e exigências impostas por treinadores, o que, de certa forma, pode comprometer o comportamento alimentar das atletas (SCHAAL et al., 2011).

Os resultados do presente estudo mostram, com relação ao sexo masculino, que esportes de equipe com bola (futebol, handebol, basquetebol), de potência (natação, atletismo) e de classe de peso (judô, tae-kwon-do) apresentaram maior prevalência de comportamento alimentar inadequado, quando comparados a modalidades de cunho estético (saltos ornamentais). Além disso, não foram encontrados atletas com comportamentos alimentares de risco em algumas modalidades de classe de peso (esgrima) e equipes com bola (polo aquático, voleibol).

Comportamentos alimentares desordenados são incomuns em homens (FILAIRE et al., 2007). Porém, Vieira et al. (2006), avaliando atletas de judô, identi- 
ficaram prevalências de sintomas de TAs de $16 \%$ e $33 \%$ em atletas adolescentes e adultos, respectivamente. Por outro lado, Filaire et al. (2007) estudaram fatores de risco para desenvolvimento de TAs e prevalência de sintomas destas psicopatologias em atletas adolescentes de ciclismo e judô do sexo masculino. Utilizando o mesmo questionário do presente estudo (EAT-26), estes autores não encontraram nenhum indivíduo com pontuação superior a 20, que é o indicativo de comportamento alimentar inadequado.

Em relação ao sexo feminino, modalidades de equipe com bola (handebol, basquetebol), de potência (natação) e de classe de peso (tae-kwon-do) apresentaram prevalência de comportamento alimentar inadequado semelhante aos esportes de características estéticas, como nado sincronizado e ginástica artística, contrapondo, então, resultados expostos em outros estudos (SCHAAL et al., 2011; HAUSENBLAS; DOWNS, 2001).

Talvez, a discrepância deste resultado em relação a outras investigações possa ser justificada pelas diferenças culturais encontradas no Brasil (FORTES et al., 2011; PERINI et al., 2009). Pesquisas deste tipo são raras na população de atletas, e mais escassas ainda comparando-se os sintomas de TAs entre diferentes modalidades esportivas. Em um estudo realizado na Europa (SCHAAL et al., 2011), comparou-se a prevalência de TAs em atletas de 36 esportes classificados em: estético, combate, alto risco, habilidades motoras finas, corrida, raquete e esportes de equipe com bola. Os autores supracitados identificaram que atletas do sexo feminino de esportes com características estéticas e de habilidades motoras finas apresentaram maior frequência de alimentação transtornada em relação às atletas das demais modalidades esportivas.

Os estudos de riscos para TAs com atletas, em sua maioria, apresentaram maiores frequências de comportamentos alimentares inadequados, quando comparados à população geral (FILAIRE et al., 2007; SUNDGOT-BORGEN; TORSTVEIT, 2004). Entretanto, isto ainda não é consenso na literatura. Apesar destas constatações, raros estudos têm comparado a prevalência de alimentação desordenada entre diferentes modalidades esportivas, na tentativa de identificar esportes que possam apresentar efetivamente maiores riscos no desenvolvimento de tais síndromes.

No Brasil, os estudos que tentaram descrever prevalências e/ou fatores de risco para o desenvolvimento destas doenças em atletas, utilizaram baixo tamanho amostral, portanto, trazendo resultados que não devem ser generalizados para a população de atletas brasileiros. Por isso, é notória a existência de um campo de estudo a ser explorado no Brasil. No entanto, recomenda-se a criação ou validação psicométrica de instrumentos com adaptação transcultural para rastrear estas psicopatologias entre os atletas.

O presente estudo apresentou algumas limitações. Uma delas foi utilizar instrumentos autoaplicáveis para rastrear riscos de determinadas doenças. Isso pode mascarar a prevalência de sintomas de tais síndromes, já que os sujeitos da 
pesquisa podem não responder com total veracidade às perguntas do questionário, pois, por se tratarem de atletas, correm o risco de serem afastados ou até cortados do processo de treinamento físico. Outra limitação foi o baixo tamanho amostral de algumas modalidades esportivas inseridas no estudo, tais como: atletismo, taekwon-do e saltos ornamentais. Contudo, ressalta-se a dificuldade em conseguir clubes e atletas que aceitem participar de forma voluntária de estudos como este.

Por fim, os resultados da presente investigação permitem presumir que atletas de basquetebol do sexo masculino estão mais suscetíveis para o desencadeamento de TAs. Em contrapartida, esportistas do sexo feminino podem estar vulneráveis para os TAs, independentemente da modalidade que praticam.

\title{
PREVALENCE OF BODY DISSATISFACTION AND EATING BEHAVIORS OF RISK FOR EATING DISORDERS IN YOUNG ATHLETES
}

\begin{abstract}
The aim of this study was to compare the prevalence of Body Dissatisfaction (BD) and Inappropriate Eating Behavior (IEB) in young athletes. 580 athletes in both sexes participated. BD was evaluated by Body Shape Questionnaire. We examined the IEB for the Eating Attitudes Test. We used the chi-square test to compare the prevalence of gender and sports. The results showed that basketball and judo were modalities with more dissatisfaction with body among male athletes $(\mathrm{p}<0,05)$. In girls, these differences were significant between some sports $(\mathrm{p}<0,05)$. In addition, boys who play soccer, basketball and tae-kwon-do showed a higher prevalence of IEB $(\mathrm{p}<0,05)$. We concluded that there are differences of prevalence of BD and IEB among sports for each sex.

Keywords: Eating disorders. Body image. Athletes.

PREVALENCIA DE INSATISFACCIÓN CORPORAL Y LA CONDUCTA ALIMENTARIA DE RIESGO PARA LOS TRANSTORNOS ALIMENTARIOS EN JÓVENES DESPORTISTAS
\end{abstract}

\section{Resumen}

El objetivo de este estudio fue comparar la prevalencia de la Insatisfacción Corporal (IC) y el Comportamiento Inadecuado de Comer (CAI) en atletas jóvenes. 580 atletas participaron en ambos sexos. IC se evaluó mediante el Body Shape Questionnaire. Hemos examinado la CAI para el Eating Attitudes Test. Se utilizó la prueba de chi-cuadrado para comparar la prevalencia de género y el deporte. Los resultados mostraron que el baloncesto y el judo fueron las modalidades que fueron los atletas más hombres con insuficiencia cardiaca $(p<0,05)$. En las niñas, estas diferencias fueron significativas entre algunos deportes $(\mathrm{p}<0,05)$. Además, los niños que juegan fútbol, básquetbol y tae-kwon-do mostró una mayor prevalencia de la IC $(\mathrm{p}<0,05)$. Se concluyó que las diferencias en la prevalencia de la IC y el CAI entre los deportes para cada sexo.

Palabras-Clave: Trastornos de la alimentación. La imagen corporal. Los atletas.

Pensar a Prática, Goiânia, v. 17, n. 1, p. 01-294, jan./mar. 2014 


\section{Referências}

BAUM, A. Eating Disorders in the Male Athlete. Sports Medicine, v.36, n.1, p.16, 2006.

BIGHETTI, F.; SANTOS, C. B.; SANTOS, J. E.; RIBEIRO, R. P. P. Tradução e avaliação do Eating Attitudes Test em adolescentes do sexo feminino de Ribeirão Preto, São Paulo. Jornal Brasileiro de Psiquatria, v.53, n.6, p.339-346, 2004.

CONTI, M. A.; CORDÁS, T. A.; LATORRE, M. R. D. O. Estudo de validade e confiabilidade da versão brasileira do body shape questionnaire (bsq) para adolescentes. Revista Brasileira de Saúde Materna e Infantil, v.9, n.3, p.331-338, 2009.

DENOMA, J. M. H.; SCARINGI, V.; GORDON, K. H. VAN ORDEN, K. A.; JOINER, T. E. Eating Disorder Symptoms among Undergraduate Varsity Athletes. Club Athletes, Independent Exercisers. And Nonoexercises. International Journal of Eating Disorders, v.12, n.1, p.47-53, 2009.

FILAIRE, E.; ROUVEIX, M.; PANNAFIEUX, C.; FERRAND, C. Eating atitudes, perfectionism and body-esteem of elite male judoists and cyclists. Journal of Sports Science and Medicine, v. 6, n.1, p.50-57, 2007.

FORTES, L. S.; AMARAL, A. C. S.; CONTI, M. A.; FERREIRA, M. E. C. Qualidades psicométricas do Eating Attitudes Test (EAT-26) para a população adolescente masculina brasileira. Psicologia: Reflexão e Crítica, no prelo.

FORTES, L. S.; FERREIRA, M. E. C. Comparação da insatisfação corporal e do comportamento alimentar inadequado em atletas adolescentes de diferentes modalidades esportivas. Revista Brasileira de Educação Física e Esporte, v.25, n.4, p.707-716, 2011.

FORTES, L. S.; MIRANDA, V. P. N.; AMARAL, A. C. S.; FERREIRA, M. E. C. Insatisfação corporal de adolescentes atletas e não atletas. Jornal Brasileiro de Psiquiatria, v.60, n.4, p.309-314, 2011.

HAUSENBLAS, H. A.; DOWNS, D. S. Comparison of Body Image between Athletes and Nonathletes - a Meta-Analytic Review. Journal Applied Sports Psychology, Philadelphia, v.13, n.2, p.323-339, 2001. 
KRENTZ, E. M.; WARSCHBURGER, P. Sports-related correlates of disordered eating in aesthetic sports. Psychology of Sport and Exercise, v.44, n.3, 315-321, 2011.

PERINI, T. A.; VIEIRA, R. S., VIGÁRIO; P. S.; OLIVEIRA, G. L.; ORNELLAS, J. S., OILVEIRA, F. P. Transtorno do comportamento alimentar em atletas de elite de nado sincronizado. Revista Brasileira de Medicina do Esporte, v.15, n.1, p.54-57, 2009.

PICKETT, T. C.; LEWIS, R. J.; CASH. T. F. Men, muscles, and body image: comparisons of competitive bodybuilders, weight trainers, and athletically active controls. British Journal of Sports Medicine, v.39, n.2, p.217-222, 2005.

REIKING, M. F.; ALEXANDER, L. E. Prevalence of disordered-eating behaviors in undergraduate female collegiate athletes and nonathletes. Journal of Athletic Training, v.40, n.1, p.47-51, 2005.

SCHAAL, K. et al. Psychological balance in high level athletes: gender-based differences and sport-specific patterns. Psychopathology and High Level Sport, v.6, n.5, p.1-9, 2011.

SCHERER, F. C. et al. Imagem corporal em adolescentes: associação com a maturação sexual e sintomas de transtornos alimentares. Jornal Brasileiro de Psiquiatria, v.59, v.3, p.198-202, 2010.

SUNDGOT-BORGEN, J.; TORSTVEIT, M. K. Prevalence of disorders in elite athletes in higher than in the general population. Clinical Journal Sport Medicine, v.14, n.1, p.25-32, 2004.

TORSTVEIT, M. K.; SUNDGOT-BORGEN, J. The female athlete triad exists in both elite athletes and controls. Medicine Science Sports and Exercise, v.37, n.4, p.1449-1459, 2005.

VIEIRA, J. L. L. et al. Distúrbios de Atitudes Alimentares e Distorção da Imagem Corporal no contexto Competitivo da Ginástica Rítmica. Revista Brasileira de Medicina do Esporte, v.15, n.6, p.410-414, 2009a.

VIEIRA, J. L. L. et al. Distúrbios de atitudes alimentares e sua relação com o crescimento físico de atletas paranaenses de Ginástica Rítmica. Motriz, Rio Claro, v.15, n.3, p.552-561, set/out, 2009b. 
VIEIRA, L. F. et al. Distúrbios de atitudes alimentares e sua relação com a distorção da auto-imagem corporal em atletas de judô do estado do Paraná. Revista da Educação Física/UEM, v.17, n.2, p.177-184, 2006.

O presente trabalho contou com apoio financeiro do Conselho Nacional de Desenvolvimento Científico e Tecnológico (CNPq).

Recebido em: 09/08/2012

Revisado em: 10/10/2012

Aprovado em: 13/01/2013

Endereço para correspondência:

caputoferreira@terra.com.br

Maria Elisa Caputo Ferreira

Universidade Federal de Juiz de Fora

Instituto de Ciências Humanas, Faculdade de Educação Física.

CAMPUS UNIVERSITÁRIO

CIDADE UNIVERSITÁRIA

Juiz de Fora, MG - Brasil 